\title{
Inflammasome gene expression alterations in Staphylococcus aureus biofilm-associated chronic rhinosinusitis*
}

\author{
Camille Jardeleza', Dijana Miljkovic', Leonie Baker', Samuel Boase', \\ Neil Cheng-Wen Tan', Simon A. Koblar², Peter Zalewski', Maureen \\ Rischmueller ${ }^{3}$, Susan Lester ${ }^{3}$, Amanda Drilling', Damien Jones'1, \\ Lor Wai Tan', Peter-John Wormald', Sarah Vreugde' \\ ' Department of Surgery-Otorhinolaryngology, Head and Neck Surgery, University of Adelaide, Adelaide, Australia \\ 2 Stroke Research Programme, C/O School of Medicine, TQEH Campus, University of Adelaide, Adelaide, Australia \\ ${ }^{3}$ Centre for Inflammatory Diseases Research, Basil Hetzel Research Institute Department of Medicine, University of Adelaide, Ade- \\ laide, Australia
}

Rhinology 51: 315-322, 2013

DOI:10.4193/Rhino13.045

* Received for publication:

April 17, 2013

Accepted: May 28, 2013

\section{Summary}

Background: The role of inflammasomes in chronic inflammation has been the subject of intense research in recent years. Chronic rhinosinusitis (CRS), a persistent inflammatory disease, continues to be investigated hoping that a clearer pathophysiologic description will guide discovery of future treatment modalities. This study investigates the role of inflammasome complexes in CRS patients with Staphylococcus aureus biofilm infection, a key culprit associated with disease severity and recalcitrance.

Methodology: Sinonasal tissue samples were collected from CRS patients with $(\mathrm{P}+)$ and without $(\mathrm{P}-)$ polyps and controls. $\mathrm{S}$. aureus biofilm status was obtained using fluorescence in situ hybridization and classified as biofilm positive (B+) or negative (B-). RNA was analysed using a Human Inflammasome PCR array, profiling the expression of 84 genes involved in inflammasome function.

Results: Sixteen samples were obtained: $5 \mathrm{~B}+\mathrm{P}+, 5 \mathrm{~B}-\mathrm{P}$ - and 6 controls. Comparing $\mathrm{B}+\mathrm{P}+\mathrm{vs}$. controls showed the greatest number of differentially expressed genes. In particular, Absent in Melanoma 2 (AIM2) was consistently and significantly up-regulated in the $\mathrm{B}+\mathrm{P}+\mathrm{vs}$. B-P- and controls. In contrast, when comparing the B-P- vs. controls, no genes showed significant changes.

Conclusion: Our results indicate the involvement of inflammasome complexes and their signalling pathways in CRS patients with polyps and S. aureus biofilms. In particular, AIM2, activated by intracellular double-stranded DNA, is up-regulated in this group, implying that $S$. aureus may play a role in intracellular triggering of the inflammasome response. Studies with further patient stratification and assessing corresponding protein expression are needed to further characterize the role of inflammasomes in CRS.

Key words: inflammasome, chronic rhinosinusitis, CRS, Staphylococcus aureus, S. aureus, biofilms, innate immunity, Absent In Melanoma 2, AIM2

\section{Introduction}

Chronic rhinosinusitis (CRS) is an inflammatory disease of the sinonasal cavity characterized by a complex interplay of the host, environment and microbe. To date, there is still no clear understanding of its pathophysiology. This may indirectly account for a myriad of treatment strategies which, although initially effective in controlling symptoms, do not completely eradicate the disease. As a result, symptom recurrence often 
occurs despite maximum medical and surgical therapy, causing a significant burden to both patient and society ${ }^{(1)}$. Further defining the relationship of the factors involved in CRS is essential, in the hope that this understanding of the underlying molecular basis of the disease will lead to developing more targeted and successful treatment modalities.

The host response to external factors such as microbes and allergens in CRS patients is one component that needs further investigation. Furthermore, in a subset of patients with greater disease severity and higher risk of recalcitrant infection, Staphylococcus aureus (S. aureus) has been identified as a key player ${ }^{(2,3)}$. Its role in initiating or sustaining an inflammatory response in CRS requires further characterization.

Several types of innate immune receptors, expressed on mucosal surfaces and hematopoietic cells, sense the presence of microbial products through receptors for pathogen-associated molecular patterns (PAMPs), resulting in the regulated production of bactericidal products and pro-inflammatory factors. These innate pattern-recognition receptors (PRRs) include Tolllike receptors (TLRs) and C-type lectin receptors, which scan the extracellular milieu and endosomal compartments for PAMPs. Within the cytosolic compartment, nucleic-acid sensing PRRs such as the RNA-sensing RIG-like helicases (RLHs), the DNA sensors, Z-DNA-binding protein (ZBP1) and Absent in Melanoma 2 (AIM2), and the Nucleotide Oligomerization Domain-like (NODlike) receptors (NLRs), cooperate to provide cytosolic surveillance and can recognize PAMPs, as well as host-derived danger signals (danger-associated molecular patterns, DAMPs) ${ }^{(4)}$. Inflammasomes are a group of cytoplasmic protein complexes containing a cytosolic PRR that react to infection and host tissue injury by activation of Caspase-1. This induces the maturation and secretion of the pro-inflammatory cytokines IL-1 $\beta$ and IL-18 ${ }^{(4)}$, and lead to cellular pyroptosis ${ }^{(5)}$. Pyroptosis involves the rapid rupture of cell membranes and spilling of pro-inflammatory intracellular contents. Uniquely dependent on Caspase-1 activity, this type of cell death promotes inflammation ${ }^{(6)}$ and is found to occur more frequently in infection with intracellular pathogens ${ }^{(7-9)}$. Thus inflammasomes have been found to play a critical role in the control of microbial infection ${ }^{(10)}$.

Inflammasomes have been the subject of intense interest in the last few years. Recently found to play a significant role in a variety of auto-inflammatory diseases such as gout and Type 2 Diabetes ${ }^{(4)}$ they have also been implicated in airway inflammation ${ }^{(11)}$ and autoimmune disorders ${ }^{(12)}$, whose chronic inflammatory nature share many similarities to CRS.

S. aureus in the planktonic and/or biofilm form plays a central role in the pathophysiology of CRS ${ }^{(13)}$. Its isolation at the time of surgery repeatedly predicts an increased risk of infection recurrence and poorer post-operative outcomes ${ }^{(14)}$. Studies have recently shown an association of bacterial biofilms with high TLR2 and Nuclear factor kappa beta (NF-k $\beta$ ) expression in sinonasal tissue samples of CRS patients ${ }^{(15)}$, indicating a role of biofilms in the innate immune response. The specific effects however, of S. aureus biofilms on the host innate immune response and inflammasome activation in the setting of CRS are still unclear. This study therefore aims to determine if $S$. aureus biofilm presence plays a role in the alternation of gene expression involved in the inflammasome pathway in the setting of CRS.

\section{Materials and methods}

Patient tissue collection and classification This study was approved by The Queen Elizabeth Hospital Human Ethics Committee and consent obtained from patients prior to surgery (Ethics number 2011057). Patients were diagnosed with CRS based on criteria outlined by the Chronic Rhinosinusitis Task Force in $2003^{(16)}$. Tissue samples were collected and categorized equally to 3 groups based on intra-operative findings: CRS with nasal polyps (CRSwNP, $n=5$ ), CRS without nasal polyps (CRSsNP, $n=5$ ) and the control (no CRS) group ( $n=6)$. CRSwNP patients must fit the criteria of having polyps visible in the middle meatus to be included in the study. Patient data such as age, past medical history, smoking history, asthma, allergies and operation type were recorded. Pre-operative symptom scores based on severity of symptoms such as nasal obstruction, postnasal drip, anterior rhinorrhea, anosmia, and facial pain/headache on a scale of 1-5, with 5 having the highest severity were recorded. Intra-operative findings such as the presence of pus, mucus and polyps were noted. Exclusion criteria were patients who were fungus positive based on culture or microscopy, as well as those on oral steroids or antibiotics within 2 weeks from the time of surgery. Tissue samples of CRSwNP patients were taken from diseased ethmoidal polypoid tissue, not focusing specifically on nasal polyps, while CRSsNP tissue samples were also taken from grossly inflamed diseased ethmoidal tissue.

Control tissue samples were obtained from non-diseased ethmoidal tissue of patients undergoing endoscopic sinus surgery (ESS) for a pituitary adenoma. Control patient CT-scans, casenotes and intra-operative reports were also reviewed prior to inclusion to ensure the absence of incidental sinus disease such as pus, mucus and polyps.

\section{Human sinonasal tissue sample collection}

Samples were collected at time of surgery and placed in Dulbecco's Modified Eagle media (Gibco, Invitrogen Life Technologies, VIC Australia) for transport. An $8 \mathrm{~mm} \times 8 \mathrm{~mm}$ tissue section was frozen and stored in $-80^{\circ} \mathrm{C}$ for subsequent biofilm analysis. Remaining tissue was stored in RNAlater (Ambion Life Techno- 
logies, Grand Island, NY, USA) for subsequent RNA extraction in preparation for the quantitative real time (qRT)-PCR array.

\section{S. aureus biofilm detection by fluorescence in situ hybridi-} zation

Fluorescence in-situ hybridization (FISH) was performed on mucosa that had been stored at $-80^{\circ} \mathrm{C}$. Defrosted samples were washed in MilliQ water prior to hybridization to remove any planktonic organisms. A S. aureus specific sequence conjugated to Alexa-488 probe was utilized (AdvanDx, Woburn, MA, USA). The manufacturer's protocol was followed. Briefly, samples were fixed to glass slides, dehydrated in $90 \%$ ethanol, air dried, and hybridized at $55^{\circ} \mathrm{C}$ for 90 minutes. Samples were scanned at 20x and 40x magnification using the Leica TCS SP5 Confocal Scanning Laser Microscope (Leica Microsystems, Wetzlar, Germany). An excitation wavelength of $488 \mathrm{~nm}$ with emission range of 495 -540nm was used to detect S. aureus. The entire sample was systematically scanned for biofilm elements. Axial Z stacks ( 0.5 $\mu \mathrm{M})$ were recorded of representative areas to construct a three dimensional virtual image of the tissue, overlying mucus and biofilm.

\section{RNA extraction method}

One hundred mg wet weight of tissue was removed from RNAlater and homogenised using a TissueRuptor (QIAGEN Pty Ltd, VIC Australia) followed by extraction of RNA using the RNeasy Lipid tissue Mini Kit according to the manufacturer's instructions (QIAGEN). An on-column DNAse treatment was performed using an RNase-Free DNase Set (QIAGEN).

\section{RNA quality / quantity}

RNA integrity was checked using an Experion RNA StdSens Kit on the Experion Electrophoresis station (BioRad Laboratories Inc, NSW Australia). RNA samples with undegraded 18 S and $28 \mathrm{~S}$ ribosomal subunit signals were used for subsequent analysis. RNA concentration was quantified using a NanoDrop 1000 Spectrophotometer (Thermo Scientific, Wilmington DE).

\section{Reverse transcription}

One thousand ng of RNA was reverse transcribed into complementary DNA (CDNA) using the RT2 First Strand Kit (SABiosciences QIAGEN Pty Ltd, VIC Australia). The incubation steps for the reaction were performed on a MyCycler Thermal Cycler (BioRad).

\section{Real time quantitative PCR array}

The cDNA sample was then combined with RT2 SYBR Green / Fluorescein qPCR Master Mix with Nuclease-free Sterile $\mathrm{H} 2 \mathrm{O}$ (QIAGEN) and then added across an entire RT2 Profiler ${ }^{\text {TM }}$ PCR Human Inflammasome Array (PAHS097, SABiosciences) 96 well plate. This consisted of a panel of 84 wells that corresponded to 84 different genes of the inflammasome pathway, 5 house keeping control wells, 1 genomic DNA control well, 3 reverse transcription control wells and three positive PCR control wells. The cDNA from each patient was amplified using an iCycler with iQ5 Software (BioRad).

\section{Statistical analysis}

Data was analysed to compare patient groups with each other using the supplied SA Biosciences $\mathrm{RT}^{2}$ Profiler PCR Array Data Analysis Template v3.3 (SABiosciences). This program automatically computes the normalized gene expression in the test samples divided by the normalized gene expression in the control samples and also computes the corresponding $\mathrm{p}$-values for each gene using the student's T-test in test and control samples.

\section{Results}

Patient demographics

Sixteen patient tissue samples were collected 5 CRSwNP S. aureus biofilm positive patient samples $(\mathrm{B}+\mathrm{P}+), 5 \mathrm{CRSsNP}$ S. aureus biofilm negative samples (B-P-), and 6 control samples. Figure 1 shows representative examples of FISH S. aureus biofilm status. There were 9 females and 7 males with an average age of 48.25 years (28 - 72 years). All control tissue samples were tested for $S$. aureus biofilm using $\mathrm{FISH}$, all of which were negative.

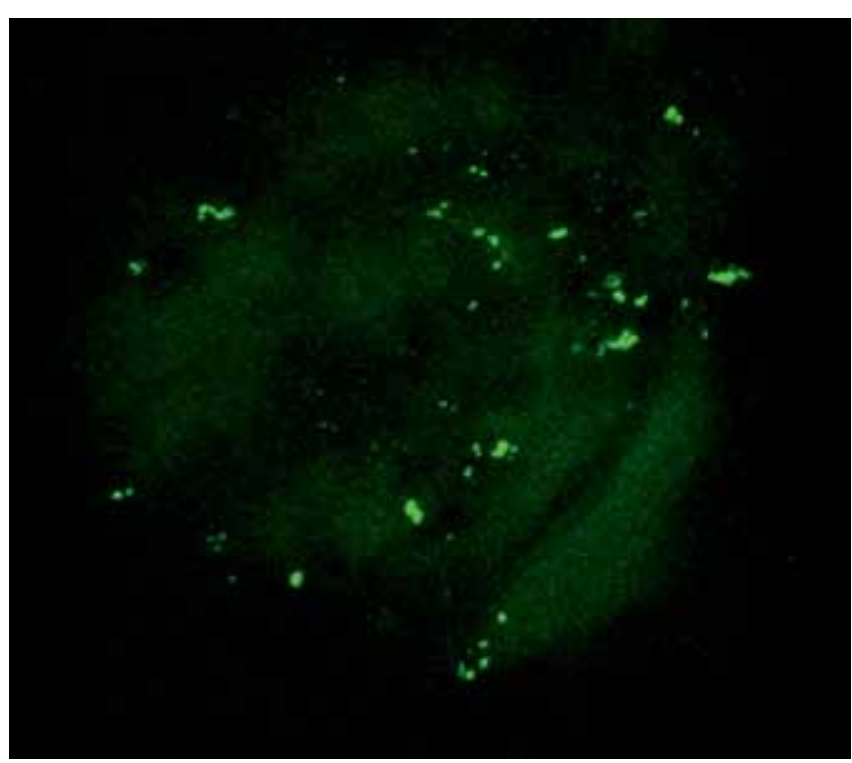

Figure 1. Confocal scanning laser microscopy image of S. aureus biofilms on the sinonasal mucosa of $\mathrm{B}+\mathrm{P}+$ patient. Note the bright green fluorescence of biofilms using a species-specific FISH probe. $\mathrm{B}+\mathrm{P}+$ : Biofilm positive polyp positive. FISH: fluorescence in-situ hybridization. 
Table 1. Summary of patient demographics and clinical findings in the 3 groups.

\begin{tabular}{|c|c|c|c|c|c|}
\hline Patient Group & $\begin{array}{c}\text { Average Age } \\
\text { (years) }\end{array}$ & M:F ratio & Revision Surgery? & $\begin{array}{l}\text { Immunocap } \\
\text { Allergy test }\end{array}$ & Culture swab results \\
\hline Control & 54.5 & 1:5 & All No & Not done & N/A \\
\hline$B+P+$ & 50.8 & $3: 2$ & All Yes & $\begin{array}{l}4 \text { positive } \\
1 \text { negative }\end{array}$ & $\begin{array}{l}\text { - } 4 \text { S. aureus } \\
\text { - } 1 \text { S. pneumoniae }\end{array}$ \\
\hline$B-P-$ & 38.2 & $3: 2$ & All No & All negative & $\begin{array}{l}\text { - } 2 \text { not sent } \\
\text { - } 1 \text { S. aureus \& } \\
\text { S. pneumoniae } \\
\text { - } 1 \text { H. influenzae } \\
\text { - } 1 \text { P. mirabilis }\end{array}$ \\
\hline
\end{tabular}

B+ P+: Biofilm positive polyp positive; B- P-: Biofilm negative polyp negative; M:F: Male:Female; S. aureus: Staphylococcus aureus; S. pneumoniae: Streptococcus pneumoniae; H. influenzae: Haemophilus influenzae; P. mirabilis: Proteus mirabilis.

\section{Clinical data}

Symptom scores were higher in the $\mathrm{B}+\mathrm{P}+$ group with an average of $19.6 \pm 1.3$ out of a total of 25 , while the B-P-group had an average symptom score of $15 \pm 3.6$. Anosmia was the most severe symptom in the $\mathrm{B}+\mathrm{P}+$ group with an average subjective score of 4.4 out of 5 , while nasal obstruction was the worst symptom in the B-P-group with an average of 3.6. All B+P+ patients had a history of previous sinus surgery and had more extensive operations compared to the B-P-group. Four out of 5 of the $\mathrm{B}+\mathrm{P}+$ patients had allergies to at least one environmental allergen. The B-P-group were all RAST negative and were all undergoing their first sinus surgery.

In the B-P-group, only 1 patient had asthma and none had Aspirin sensitivity. Three out of 5 patients in the $B+P+$ group had asthma, one of which also had Aspirin sensitivity (Samter's triad). None of the control patients were asthmatics nor had Aspirin sensitivity (Table 1).

Expression of AIM2 inflammasome complex and associated pathway proteins

In the $\mathrm{B}+\mathrm{P}+$ patient group, there was a significant fold increase in the mRNA expression of AIM2 ( $p$-value $0.004,+3.17$ ), CASP5 (Caspase-5), CCL2 (Chemokine Ligand 2), IFN- $\gamma$ (Interferon- $\gamma$ ), IL-1 $\beta$ (Interleukin 1 $\beta$ ) and RANKL (Receptor activator of nuclear factor kappa- $\beta$ ligand) compared to the control group. There were corresponding significant fold reductions in XIAP (X-linked inhibitor of apoptosis protein), HSP90AA1 (Heat shock Protein $90 \mathrm{KDa}$ a) and PANX1 (Pannexin 1) mRNA. Table 2 summarizes these significant gene changes.

By contrast, differences in mRNA expression between the B-Ppatient category when compared to non-diseased control sinonasal mucosa were unremarkable with none of the genes recor- ding a significant, more than 2-fold up- or down-regulation.

When comparing the $\mathrm{B}+\mathrm{P}+$ vs the $\mathrm{B}-\mathrm{P}$ - groups, the $\mathrm{B}+\mathrm{P}+$ group showed a significant up-fold regulation of AIM2 ( $p$-value 0.02, +2.57 ) as well as CCL2, RANKL, PSTPIP1 (proline-serine- threonine phosphatase interacting protein 1) and PYCARD (PYD CARD Domain containing). There was a significant down-fold regulation of only 1 gene, PANX1 ( $p$-value $0.03,-2.23$ ). Table 3 summarizes the gene changes when comparing these groups.

When looking at the asthmatic $\mathrm{B}+\mathrm{P}+$ patients vs. the controls, the number of genes significantly up-regulated dropped to 3 : AIM2, CCL2 and TNFSF11 (Tumor necrosis factor member 11, p-value $0.0001,+4.98)$. Grouping all patients with asthma regardless of biofilm and polyp status showed no genes significantly altered when compared to controls. This included $1 \mathrm{~B}-\mathrm{P}$ - and 3 $\mathrm{B}+\mathrm{P}+$ patients.

Only 4 genes were found to be consistently and significantly altered when comparing patient groups based on biofilm and polyp status: AIM2, CCL2 and RANKL, which were significantly up-regulated, and PANX1, which was significantly down-regulated in the $\mathrm{B}+\mathrm{P}+$ group when compared to $\mathrm{B}-\mathrm{P}$ - and controls.

\section{Discussion}

The results of our study show that an involvement of the inflammasome signalling pathway, with an up-regulation of AIM2, CCL2 and IL1 $\beta$, exists in CRS in the presence of nasal polyps and $S$. aureus biofilms, indicating an increased inflammatory potential in this subset of patients. The up-regulation of AIM2 in particular, has potential implications in the pathophysiologic process of S. aureus-associated infection in CRS. AIM2, recently identified as a member of the inflammasome family, was found 
Table 2. Expression of inflammasome related genes in sinonasal tissue harvested from $B+P+$ patients compared with non-diseased control mucosa and their biological functions.

\begin{tabular}{|c|c|c|c|c|}
\hline Gene & Gene Name & Biological function & Fold change & p value \\
\hline AlM2 & Absent in Melanoma 2 & $\begin{array}{l}\text { The C-terminal HIN domain of AIM2 binds double stranded viral, bacte- } \\
\text { rial, or host DNA and acts as a cytosolic double stranded DNA sensor }\end{array}$ & +3.17 & 0.005 \\
\hline CASP5 & Caspase-5 & $\begin{array}{l}\text { Part of the NLRP1 inflammasome complex. It potentiates Caspase-1 } \\
\text { activity and involved in the generation of IL-1 } \beta \text {. }\end{array}$ & +9.91 & 0.049 \\
\hline $\mathrm{CCL} 2$ & Chemokine Ligand 2 & $\begin{array}{l}\text { A small cytokine which recruits monocytes, memory } T \text { cells, and den- } \\
\text { dritic cells to sites of injury, infection, and inflammation. It has been } \\
\text { implicated in the pathogenesis of diseases characterized by monocytic } \\
\text { infiltrates, like psoriasis, rheumatoid arthritis and atherosclerosis. }\end{array}$ & +4.71 & 0.005 \\
\hline IFN- $\gamma$ & Interferon- $\gamma$ & $\begin{array}{l}\text { Has antiviral, immunoregulatory, and anti-tumor properties. It increases } \\
\text { lysosome activity in macrophages. Also has the ability to degrade the } \\
\text { RANK adaptor protein TRAF6 (tumor necrosis factor receptor-associated } \\
\text { factor } 6 \text { ) in the RANK-RANKL signalling pathway, which otherwise stimu- } \\
\text { lates the production of NF-KB. }\end{array}$ & +2.22 & 0.014 \\
\hline IL-1 $\beta$ & Interleukin $1 \beta$ & $\begin{array}{l}\text { Pro-inflammatory cytokine generating local response to tissue injury and } \\
\text { infection. Promotes leukocyte infiltration at the site of injury, activates } \\
\text { lymphocytes. }\end{array}$ & +2.62 & 0.04 \\
\hline RANKL & $\begin{array}{l}\text { Receptor activator of } \\
\text { nuclear factor kappa- } \beta \\
\text { ligand }\end{array}$ & $\begin{array}{l}\text { Activates anti apoptotic kinase AKT/PKB through a signalling complex } \\
\text { involving SRC kinase and TRAF6. It may have a role in the regulation of } \\
\text { cell apoptosis. }\end{array}$ & +5.26 & 0.015 \\
\hline XIAP & $\begin{array}{l}\text { X-linked inhibitor of } \\
\text { apoptosis protein }\end{array}$ & $\begin{array}{c}\text { Stops apoptosis induced either by viral infection or by Caspase } \\
\text { overproduction }\end{array}$ & -2.41 & 0.034 \\
\hline HSP90AA1 & $\begin{array}{l}\text { Heat shock Protein } 90 \\
\text { KDa a }\end{array}$ & $\begin{array}{l}\text { Cytosolic inducible heat shock protein. Produced in response to } \\
\text { environmental stress including heat; protects against cellular stress }\end{array}$ & -3.01 & 0.022 \\
\hline PANX1 & Pannexin 1 & $\begin{array}{l}\text { Recruited when } \mathrm{P} 2 \mathrm{X} 7 \text { is activated. It is required for Caspase- } 1 \text { activation } \\
\text { in response to ATP, nigericin and maitotoxin }\end{array}$ & -2.01 & 0.02 \\
\hline
\end{tabular}

$\mathrm{M}=$ Male; $\mathrm{F}=$ Female; $\mathrm{BMI}=$ Body mass index; $\mathrm{IIH}=$ Idiopathic intracranial hypertension.

Table 2. Summary of genes significantly changed upon comparison of the 3 groups.

\begin{tabular}{lrlll}
\hline B+P+ vs. Control & & B+P+ vs. B-P- & B-P- vs. Control \\
& & & & Nil \\
AIM2 & $\uparrow$ & AIM2 & $\uparrow$ & \\
CASP5 & $\uparrow$ & CCL2 & $\uparrow$ & \\
CCL2 & $\uparrow$ & RANKL & $\uparrow$ & \\
IFN- - & $\uparrow$ & PSTPIP1 & $\uparrow$ & \\
IL1- $\beta$ & $\uparrow$ & PYCARD & $\uparrow$ & \\
RANKL & $\uparrow$ & PANX1 & $\downarrow$ \\
XIAP & $\uparrow$ & & \\
HSP90AA1 1 & & & \\
PANX1 & $\downarrow$ & &
\end{tabular}

$\mathrm{B}+\mathrm{P}+$ : Biofilm positive polyp positive; $\mathrm{B}-\mathrm{P}-$-: Biofilm negative polyp negative. Gene abbreviations as in previous table; PSTPIP1: Proline-serinethreonine phosphatase interacting protein. PYCARD: PYD CARD Domain containing. $\uparrow$ : gene up-regulated; $\downarrow$ : gene down-regulated. $P$ value $<$ 0.05 . to be the elusive detector of cytoplasmic double-stranded DNA (dsDNA) originating from bacteria, viruses or from the host itself (17). Binding of AIM2 to foreign DNA ligand in the cell engages and recruits the Apoptosis-associated Speck-like protein with a CARD (ASC) through its pyrin domain to activate Caspase-1. This induces the maturation of pro-IL-18 and pro-IL-1 $\beta$ and may lead to pyroptotic cell death ${ }^{(18)}$ and further inflammation. This identifies AIM2 as an important sensor of potentially damaging intracellular DNA from either microbial or viral infections or tissue damage, initiating a pro-inflammatory innate immune response and contributing to the persistent inflammation that characterizes the disease process.

It is well known that $S$. aureus biofilms play an important role in eliciting or at least sustaining mucosal inflammation particularly in CRS patients with recalcitrant disease, and that the presence of S. aureus biofilms correlates with intramucosal S. aureus infection in CRS ${ }^{(19)}$. Therefore, it may be that inflammasome activation with increased IL-1 $\beta$ and AIM2 expression in the $B+P+$ groups 
may be related to the intracellular presence of S. aureus inciting a host innate immune response. This may be triggered either by direct engagement of the AIM2 protein with S. aureus DNA, or, alternatively, by molecules released upon mucosal damage. If proven true, this contradicts previous discussions that intramucosal or intracellular bacteria modulate and evade the host immune response in order to serve as a recurrent nidus of infection (20,21). Alpha-hemolysin, a major cytotoxic agent produced by $S$. aureus, has already been shown to activate the inflammasome through the NOD-like receptor pyrin domain containing protein (NLRP3) ${ }^{(22,23)}$. It is important to note however, that the absence of stratified groups separating biofilm and polyp status in this study means that the exact contribution of S. aureus biofilms or nasal polyps in the observed gene expression alterations cannot be definitively made. In a study by Mansson et al. ${ }^{(24)}$, it has been demonstrated that expression of inflammasomes comprising NLRP3 and other NLRs such as NOD1 and NOD2 are increased in nasal polyps compared to healthy nasal mucosa. This may reflect the presence of inflammatory infiltrating cells in nasal polyposis. It remains to be seen if AIM2 up-regulation is present in CRS polyp patients without $S$. aureus biofilms. Further patient stratification is required to address this issue. Patients with polyps without biofilm formation are very rare and consequently not enough patients were able to be recruited into this category during the study period. This also holds true for the biofilm positive polyp negative group.

The significant up-regulation of IL- $1 \beta$ in the $B+P+$ group may prove to be an important finding. IL-1 $\beta$ is a potent pro-inflammatory mediator that coordinates inflammatory cell recruitment to sites of infection or tissue injury and is also elevated in a variety of other inflammatory conditions such as rheumatoid arthritis ${ }^{(25)}$. The activity of IL- $1 \beta$ is tightly controlled by expression, maturation and secretion, and further research will be needed to determine whether the increased IL-1 $\beta$ mRNA expression in this subset of patients eventually results in increased IL-1 $\beta$ secretion and activity.

The pro-inflammatory Caspase-5 (+9.91 fold, $p=0.049$ ), which is part of the NLRP1 inflammasome, was also shown to be upregulated in the $\mathrm{B}+\mathrm{P}+$ group. Since it's discovery over 15 years ago ${ }^{\text {(26) }}$ not much is known about Caspase-5 except that it is part of the NLRP1 inflammasome that potentiates the activity of Caspase-1, by involvement in the generation of IL-1 $\beta^{(27)}$. It can be activated by lipopolysaccharide and interferon- $\gamma^{(28)}$ and is found to be significantly overexpressed in psoriatic skin lesions ${ }^{(29)}$.

Up-regulation of molecules involved in downstream signalling as shown by CCL2 expression ( +4.71 fold, $p=0.005$ ) lends support that circulating sentinel cells such as monocytes, macrophages and antigen-presenting cells are recruited to the site of local mucosal inflammation in the $\mathrm{B}+\mathrm{P}+$ group. Specifically involved in recruiting monocytes and T-helper cells, there is substantial evidence that CCL2 has function in the regulation of both innate and adaptive immunity. CCL2 has also been found to play an important role in certain inflammatory diseases such as atherosclerosis and multiple sclerosis ${ }^{(30)}$, the nature of which share many similarities to CRS.

RANKL ( +5.26 fold, $p=0.015$ ) on the other hand, has a role in the regulation of dendritic cell function and the activation of T-cells (31). Its function in stimulating osteoclast activity and differentiation, hence enhancing bone resorption, is associated with an anti-apoptotic function ${ }^{\left({ }^{22}\right)}$. Its up-regulation in the $\mathrm{B}+\mathrm{P}+$ patients could be interpreted as a host-mediated response in reducing sinonasal tissue damage. In this case, this may prove counter productive as the host cells now become a pathogen reservoir that escapes immune surveillance. As pyroptosis is a bacterial stimulated type of programmed host cell death, a highly significant increase in RANKL therefore could represent a feedback mechanism to modulate the degree of pathogen-induced cell death. Alternatively, this can lead to excessive collateral damage as a result of intracellular AIM2-driven inflammasome signalling.

PANX1, showing a fold decrease of $-2.28(p=0.015)$ hints at receptor-ligand interactions of the NLRP inflammasome family. PANX1 aids in the extracellular release of ATP and binding of this ligand to certain surface receptors such as $\mathrm{P} 2 \mathrm{X} 7 \mathrm{is} \mathrm{known}$ to initiate recruitment of NLRP1 and NLRP3 inflammasome components ${ }^{(33)}$. However, we did not detect any significant fold changes in expression levels of the NLRP family members.

Lastly, comparing asthmatic patients with controls showed either a drop in the number of inflammasome genes significantly altered or the absence of any gene changes. This does not correlate to current findings linking the role of inflammasomes in asthma. Recently, asthma has been linked to the NLRP3/ASC inflammasome complex with evidence that this complex plays a role in airway inflammation associated with the disease ${ }^{(34)}$. The absence of these findings in this study is likely due to small patient numbers between comparison groups. The presence of only 1 patient with Aspirin sensitivity, for example, means that this factor's independent effect on inflammasome gene regulation could not be determined in this study. Separate experiments focusing on asthma and Samters triad with greater patient numbers are required to determine their effects on gene expression in CRS patients.

The current literature on the role of inflammasomes in CRS is sparse with only a few reports characterizing NLR expression in the upper airways. Bogefors et al. ${ }^{(35)}$ located NOD1, NOD2 and NLRP3 in cultured human sinonasal epithelium and reported 
an associated down-regulation of NOD1 and NLRP3 in allergic rhinitis patients during the pollen season. They also investigated the mRNA and protein expression profiles of NOD1, NOD2 and NLRP3 in diseased upper airway tissue (tonsils, adenoids and nasal polyps). Of note was that NLR mRNA expression was significantly higher in nasal polyp when compared to healthy mucosa ${ }^{(24)}$. However, the role of the AIM2 inflammasome in CRS seems to have not yet been addressed. It will be interesting to completely characterize the inflammasome signalling pathway and determine how differential expression of its key members contributes to the development of CRS. Enhancing our understanding of alternative pathways activated in mucosal inflammation and the role microbial pathogens play in these processes, is essential in elucidating molecular mechanisms of CRS aetiopathogenesis, and determining the extent bacterial presence plays in the sinonasal manifestations of the disease.

\section{Conclusion}

To our knowledge this is the first study identifying the increased expression of the AIM2 inflammasome in the presence of $S$. aureus biofilms and nasal polyposis in the setting of CRS. Future studies looking at individual effects of biofilms and nasal polyposis with greater patient numbers and stratification are re- quired in continuing the investigation of the role this molecular complex plays in this disease. With this in mind, more successful treatment strategies may emerge once a better understanding of the pathophysiology of mucosal inflammation in CRS occurs.

\section{Acknowledgement}

Garnett Passe and Rodney Williams Memorial Foundation for financial support. The Hospital Research Foundation South Australia.

\section{Authorship contribution}
$\mathrm{CJ}$ : writing, tissue collection, collating of data
DM: patient classification, experiments
$L B, D J, A D$ : real time $P C R$, experimental troubleshooting
SB, NCWT: blinded FISH assessment
SAK, MR, PZ, SL: concepts, collaboration
LWT, PJW, SV: senior authors, concepts, manuscript finalization, supervision

\section{Conflict of interest}

PJW receives royalties from Medtronic for instruments designed and is a consultant for Neilmed Pty Ltd but this is not relevant to this study.

\section{References}

1. Bhattacharyya N. Incremental health care utilization and expenditures for chronic rhinosinusitis in the United States. Ann Otol Rhinol Laryngol. 2011; 120: 423-427.

2. Foreman A, Wormald PJ. Different biofilms, different disease? A clinical outcomes study. Laryngoscope. 2010; 120: 1701-1706.

3. Singhal D, Foreman A, Bardy JJ, et al. Staphylococcus aureus biofilms: Nemesis of endoscopic sinus surgery. Laryngoscope. 2011; 121: 1578-1583.

4. Schroder K, Tschopp J. The inflammasomes. Cell. 2010; 140: 821-832.

5. Fink SL, Cookson BT. Apoptosis, pyroptosis, and necrosis: mechanistic description of dead and dying eukaryotic cells. Infect Immun. 2005; 73: 1907-1916.

6. Bergsbaken T, Fink SL, Cookson BT. Pyroptosis: host cell death and inflammation. Nat Rev Microbiol. 2009; 7: 99-109.

7. Case CL, Shin S, Roy CR. Asc and Ipaf Inflammasomes direct distinct pathways for caspase-1 activation in response to Legionella pneumophila. Infect Immun. 2009; 77: 1981-1991.

8. Fink SL, Bergsbaken T, Cookson BT. Anthrax lethal toxin and Salmonella elicit the common cell death pathway of caspase-1-dependent pyroptosis via distinct mechanisms. Proc Natl Acad Sci U S A. 2008; 105: 4312-4317.

9. Suzuki T, Franchi L, Toma C, et al. Differential regulation of caspase-1 activation, pyrop- tosis, and autophagy via Ipaf and ASC in Shigella-infected macrophages. PLoS Pathog. 2007; 3:e111.

10. Kim S, Bauernfeind F, Ablasser A, et al. Listeria monocytogenes is sensed by the NLRP3 and AIM2 inflammasome. Eur J Immunol. 2010; 40: 1545-1551.

11. Tran HB, Lewis MD, Tan LW, et al. Immunolocalization of NLRP3 Inflammasome in Normal Murine Airway Epithelium and Changes following Induction of Ovalbumin-Induced Airway Inflammation. J Allergy. 2012; 2012: 819176.

12. Shaw PJ, McDermott MF, Kanneganti TD. Inflammasomes and autoimmunity. Trends Mol Med 2011; 17: 57-64.

13. Foreman A, Singhal D, Psaltis AJ, et al. Targeted imaging modality selection for bacterial biofilms in chronic rhinosinusitis. Laryngoscope. 2010; 120: 427-431.

14. Jervis-Bardy J, Foreman A, Field J, et al. Impaired mucosal healing and infection associated with Staphylococcus aureus after endoscopic sinus surgery. Am J Rhinol Allergy. 2009; 23: 549-552.

15. Sun $Y$, Zhou B, Wang $C$, et al. Biofilm formation and Toll-like receptor 2, Toll-like receptor 4, and NF-kappaB expression in sinus tissues of patients with chronic rhinosinusitis. Am J Rhinol Allergy. 2012; 26: 104-109.

16. Benninger MS, Ferguson BJ, Hadley JA, et al. Adult chronic rhinosinusitis: definitions, diagnosis, epidemiology, and pathophysiology. Otolaryngol Head Neck Surg. 2003;
129:S1-32.

17. Schroder K, Muruve DA, Tschopp J. Innate immunity: cytoplasmic DNA sensing by the AIM2 inflammasome. Curr Biol. 2009; 19: R262-265.

18. Fernandes-Alnemri T, Yu JW, Datta P, et al. AIM2 activates the inflammasome and cell death in response to cytoplasmic DNA. Nature. 2009; 458: 509-513.

19. Tan NC, Foreman A, Jardeleza C, et al. The multiplicity of Staphylococcus aureus in chronic rhinosinusitis: correlating surface biofilm and intracellular residence. Laryngoscope. 2012; 122: 1655-1660.

20. Wood AJ, Fraser JD, Swift S, et al. Intramucosal bacterial microcolonies exist in chronic rhinosinusitis without inducing a local immune response. Am J Rhinol Allergy. 2012; 26: 265-270.

21. Sendi P, Proctor RA. Staphylococcus aureus as an intracellular pathogen: the role of small colony variants. Trends in microbiology. 2009; 17: 54-58.

22. Mariathasan S, Weiss DS, Newton K, et al. Cryopyrin activates the inflammasome in response to toxins and ATP. Nature. 2006; 440: 228-232.

23. Craven RR, Gao X, Allen IC, et al. Staphylococcus aureus alpha-hemolysin activates the NLRP3-inflammasome in human and mouse monocytic cells. PLoS One. 2009; 4: e7446.

24. Mansson A, Bogefors J, Cervin A, et al. NODlike receptors in the human upper airways: 
a potential role in nasal polyposis. Allergy. 2011; 66: 621-628.

25. Dinarello CA. Interleukin-1 beta. Crit Care Med. 2005; 33: S460-462.

26. Munday NA, Vaillancourt JP, Ali A, et al. Molecular cloning and pro-apoptotic activity of ICErelll and ICErellII, members of the ICE/CED-3 family of cysteine proteases. J Biol Chem. 1995; 270: 15870-15876.

27. Martinon F, Burns K, Tschopp J. The inflammasome: a molecular platform triggering activation of inflammatory caspases and processing of proll-beta. Mol Cell. 2002; 10: 417-426.

28. Lin $X Y$, Choi MS, Porter AG. Expression analysis of the human caspase-1 subfamily reveals specific regulation of the CASP5 gene by lipopolysaccharide and interferon-gamma. J Biol Chem. 2000; 275: 3992039926.

29. Salskov-Iversen ML, Johansen C, Kragballe $\mathrm{K}$, et al. Caspase-5 expression is upregulated in lesional psoriatic skin. J invest Dermatol.
2011; 131: 670-676.

30. Daly C, and Rollins BJ. Monocyte chemoattractant protein-1 (CCL2) in inflammatory disease and adaptive immunity: therapeutic opportunities and controversies. Microcirculation. 2003; 10: 247-257.

31. Wu L, Lin JH, Bao K, et al. In vitro effects of erythromycin on RANKL and nuclear factorkappa B by human TNF-alpha stimulated Jurkat cells. Int immunopharmacol. 2009; 9: 1105-1109.

32. Bharti AC, Aggarwal BB. Ranking the role of RANK ligand in apoptosis. Apoptosis. 2004; 9: 677-690.

33. Bernier LP. Purinergic regulation of inflammasome activation after central nervous system injury. J Gen Physiol. 2012; 140: 571 575.

34. Birrell MA, Eltom S. The role of the NLRP3 inflammasome in the pathogenesis of airway disease. Pharmacol Ther. 2011; 130: 364-370.

35. Bogefors J, Rydberg C, Uddman R, et al.
Nod1, Nod2 and Nalp3 receptors, new potential targets in treatment of allergic rhinitis? Allergy. 2010; 65: 1222-1226.

Prof. PJ Wormald

Department of Otorhinolaryngology, Head and Neck Surgery

The Queen Elizabeth Hospital 28, Woodville Road Woodville, SA 5011 Australia

Tel: $+61-88222-7158$

FAX: +61-88222-7419

E-mail: peterj.wormald@adelaide. edu.au

\section{ADVERTISEMENT}

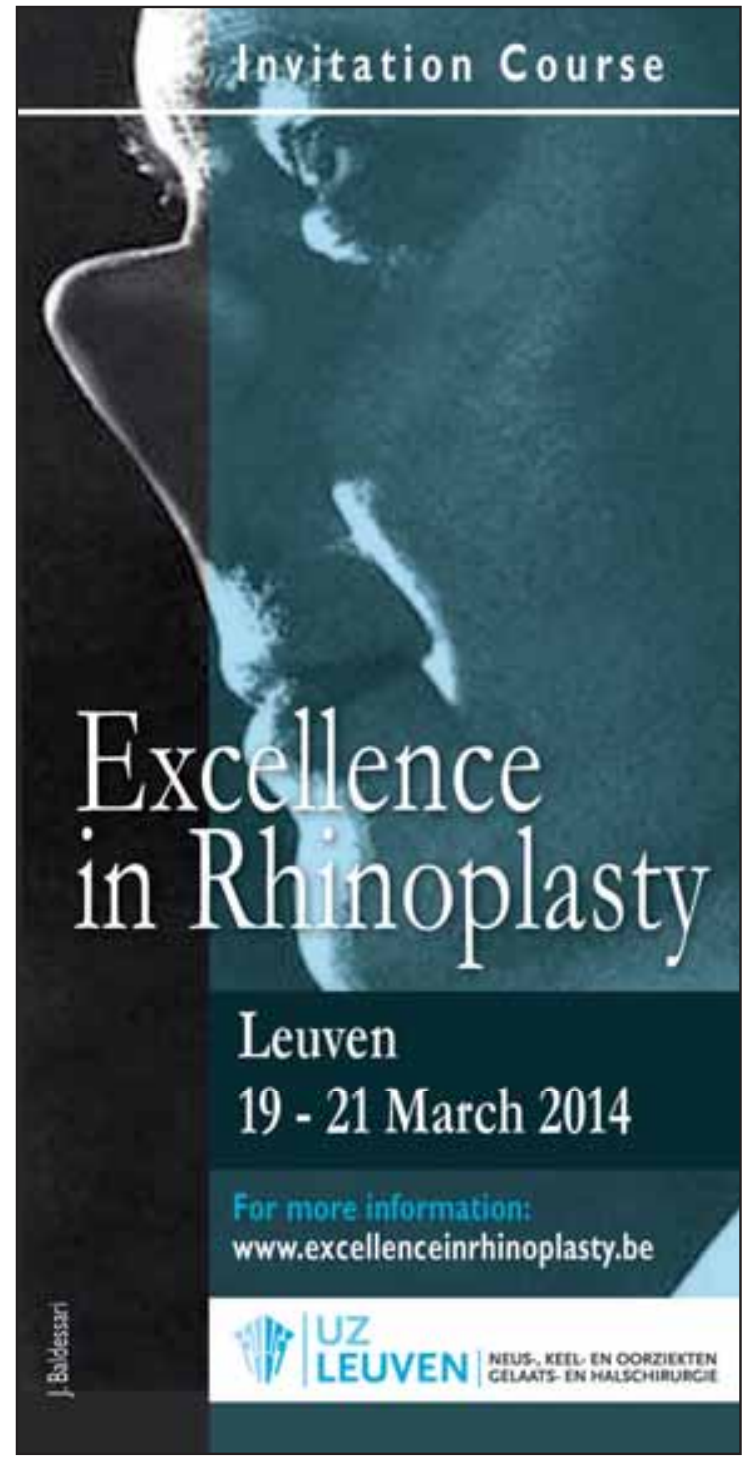

